\title{
Exercise-induced bronchoconstriction in Tunisian elite athletes is underdiagnosed
}

This article was published in the following Dove Press journal:

Open Access Journal of Sports Medicine

I June 201 I

Number of times this article has been viewed

\author{
Ridha Sallaouil-3 \\ Ines Zendah ${ }^{2}$ \\ Habib Ghedira ${ }^{2}$ \\ Mohcine Belhaouz ${ }^{3}$ \\ Mourad Ghrairi ${ }^{3}$ \\ Mohamed $\mathrm{Amri}^{3}$ \\ 'Issep Sfax, Unité de Recherche \\ "Les déterminants psychoculturels \\ et biologiques de l'accès à la \\ haute performance sportive," Sfax; \\ 2Department of Lung Function \\ Testing, Abderrahmen Mami Pneumo- \\ Allergology Hospital, Department \\ III, Tunis, Tunisia; ${ }^{3}$ Laboratoire de \\ Physiologie de la Nutrition, Faculté \\ des Sciences de Tunis, El Manar 1060 \\ Tunis, Tunisia
}

\begin{abstract}
Many studies have shown an increased risk of developing exercise-induced bronchoconstriction among the athletic population, particularly at the elite level. Subjective methods for assessing exercise-induced bronchoconstriction such as surveys and questionnaires have been used but have resulted in an underestimation of the prevalence of airway dysfunction when compared with objective measurements. The aim of the present study was to compare the prevalence of exercise-induced bronchoconstriction among Tunisian elite athletes obtained using an objective method with that using a subjective method, and to discuss the possible causes and implications of the observed discrepancy. As the objective method we used spirometry before and after exercise and for the subjective approach we used a medical history questionnaire. All of the recruited 107 elite athletes responded to the questionnaire about respiratory symptoms and medical history and underwent a resting spirometry testing before and after exercise. Post-exercise spirometry revealed the presence of exercise-induced bronchoconstriction in $14(13 \%)$ of the elite athletes, while only $1.8 \%$ reported having previously been diagnosed with asthma. In conclusion, our findings indicate that medical history-based diagnoses of exercise-induced bronchoconstriction lead to underestimations of true sufferers.
\end{abstract}

Keywords: exercise-induced bronchoconstriction, elite athletes, self-reported asthma

\section{Introduction}

The risk of developing exercise-induced bronchoconstriction (EIB) is increased among athletes, particularly those practicing at the elite level. ${ }^{1-5}$ The exact mechanisms behind this are still under debate, however the intensity of physical activity and atopy in athletes seem to be the main factors. ${ }^{5}$ The diagnosis of EIB in athletes is made according to reported symptoms, physical examination and the variability of lung function spontaneously. ${ }^{6}$ Competing athletes frequently report the presence of respiratory symptoms in relation to exercise. Currently questionnaires and surveys remain the most frequently used method of assessment. The data collected by these methods may not however always accurately reflect the respiratory condition of the participating athlete. Indeed, self-reported asthma, physician, diagnosed EIBand respiratory symptoms, all of which are frequently recorded in surveys and questionnaires, have the disadvantage of relying on subjective perception. ${ }^{7}$ Among the objective methods for evaluating the respiratory condition of athletes, the most common is the change in the forced expiratory volume in the first second $\left(\mathrm{FEV}_{1}\right)$ before and after exercise. A fall of more than $10 \%$ or $15 \%$ in $\mathrm{FEV}_{1}$ after exercise is one proposed indicator of EIB. ${ }^{7}$
Correspondence: Ridha Sallaoui

Cite Hedi Nouira Narjess B23,

2073, Tunis

Tel +21670688588

$\mathrm{Fax}+21674278502$

Email rida.sallaoui@laposte.net 
The aim of the present study was to compare the incidence of exercise-induced bronchoconstriction among Tunisian elite athletes by using the objective method of spirometry before and after exercise, and a subjective method consisting of a medical history questionnaire, and to discuss the possible causes and implications of any discrepancy.

\section{Methods}

This study was conducted over 10 different testing sessions. The tests were performed in the morning (between 9 am and $11 \mathrm{am}$ ); the mean temperature and relative humidity were $10 \pm 4^{\circ} \mathrm{C}$ (range $6-14^{\circ} \mathrm{C}$ ) and $45.6 \pm 12 \%$ (range $38 \%-55 \%)$.

Prior to exercise, a familiarization session was conducted to collect demographic information via a questionnaire, give information on the study protocol and the use of spirometry, and to introduce subjects to the investigators.

\section{Subjects}

The 107 elite athletes ( 63 males and 44 females, mean age $20.6 \pm 1.8$ years; range $17-23$ years, see Table 1 ) were divided into groups according to the type of training environment: 42 athletes trained and competed outdoors and 65 trained and competed indoors. No subjects reported upper-respiratory tract infection at the time of the study. At that time, they had been involved in their current sport for a mean of 8.5 years (range $7-11$ years) and trained regularly (mean weekly training duration: $18.3 \pm 1.9$ hours per week, range 16-21 hours per week). The outdoors activities included: soccer $(n=12)$, tennis $(n=4)$, and athletics $(n=26)$; high jump, decathlon, javelin, and middle to long-distance runners (1500 $\mathrm{m}$ to marathon). The indoor activities were: weightlifting $(n=7)$, taekwondo $(n=10)$, judo $(n=12)$, handball $(n=14)$, basketball $(n=15)$, and gymnastics $(n=7)$.

Athletes enrolled in the present study were informed in detail about the protocol and the aims of the study. Each

Table I Mean \pm SD anthropometric and spirometric variables of the studied group

\begin{tabular}{lll}
\hline Characteristics & Means \pm SD & Range \\
\hline Number of athletes & 107 & \\
Age (years) & $20.6 \pm 1.8$ & $17-23$ \\
Height (cm) & $170 \pm 10$ & $159-191$ \\
Body mass (kg) & $61.3 \pm 6.8$ & $48-75$ \\
FEV (L) & $4.0 \pm 0.7$ & $2.66-5.28$ \\
Relative value of FEV $(\%)$ & $124 \pm 14$ & $85-149$ \\
FEF25-75 (\%) & $88.7 \pm 11$ & $76-118$ \\
\hline
\end{tabular}

Abbreviations: FEF25-75, forced expiratory flow through the midportion of vital capacity; $\mathrm{FEV}_{1}$, forced expiratory volume in the one second; relative value of $\mathrm{FEV}_{1}$, percentage of theoretical values: ${ }^{26} \mathrm{SD}$, standard deviation. participant responded to a questionnaire about respiratory function and underwent a spirometry test at rest and after 8 minutes running. The research protocol, which was in accordance with the declaration of Helsinki, was approved by the Research Ethics Committee of the Faculty of Medicine, University of Sousse, Tunisia.

\section{Questionnaire: symptoms and medical history}

All athletes were asked to complete a medical history questionnaire administered in French. The questionnaire was designed by the United States Olympic Committee (USOC) Sports Medicine Division ${ }^{8}$ and was translated from English to French by an epidemiology expert before being further verified by back-translation from French to English by an independent researcher. It was the same as that used by Weiler et $\mathrm{al}^{8}$ to determine how many of the US athletes who participated in the 1998 Olympic Winter Games in Nagano and in the 1996 Olympic summer Games in Atlanta had a previous history of asthma, had symptoms suggestive of asthma, or had indicated taking a medication used to treat asthma.

\section{FEV, measurements: lung function test}

Lung function tests were carried out on a portable spirometer (Auto Spiro Pal; Minato Medical Science Co, Osaka, Japan). The athletes performed the baseline test three times and the highest result was taken into consideration. The subjects were seated comfortably; they were instructed to take a full breath in, then to close the lips around the mouth tube and blow out as hard and fast as possible. Inspiration had to be full and unhurried, and tested expiration had to be continued without pause. The technique was demonstrated to every subject and the result was expressed in liters per second. The subjects were diagnosed with asthma if any of the post-exercise $\mathrm{FEV}_{1}$ values was at least $15 \%$ lower than the pre-exercise $\mathrm{FEV}_{1}$ measurement. This level of $15 \%$ was chosen according to the recommendations of the American Thoracic Society suggesting that this level is optimal for outdoor conditions. ${ }^{9}$

$\mathrm{FEV}_{1}$ was measured at $0,5,10,15,20$, and 30 minutes after completion of exercise.

\section{Exercise performance}

The athletes ran for 8 minutes at $80 \%-85 \%$ of the estimated maximum heart rate (HRmaxTheo).

The exercise was performed without any prior warm-up and subjects ran in groups of four around an outdoor track. 
Throughout the run, subjects were equipped with portable heart rate monitors (Polar S610, Oy, Kempele, Finland) set to record the heart rate (HR) at 5-second intervals. Before the session, each subject was informed of the range of HR at which to run according to the HRmaxTheo, calculated as described by Crapo et $\mathrm{al}^{10}$ : HRmaxTheo $=(220$-age $)$.

\section{Statistical analysis}

Results concerning respiratory symptoms and nociceptive sensations were expressed as the number and percentage of athletes. Number of years of training and hours of training per week were expressed as mean \pm standard deviation (SD).

A Chi-square test was used to analyze the data from the questionnaire. Student's $t$-test for independent samples was used to determine the differences among the means of group variables. Sensitivity and specificity were calculated for the questionnaire to identify EIB, and the kappa coefficient was also calculated to show the degree of agreement. Statistical significance was fixed at the $P<0.05$ level.

\section{Results}

All of the recruited 107 athletes (mean age $20.6 \pm 1.8$ years; range 17-23 years) completed the questionnaire and the spirometric and running tests. The clinical characteristics of the study groups are given in Table 1 .

Post-exercise spirometry tests revealed the presence of EIB in $13 \%$ of the athletes (14 out of the 107). When individual data for each subject were pooled and analyzed by parametric statistical analysis, no significant intergroup differences were observed in demographic variables or in pre-exercise $\mathrm{FEV}_{1}$ (Table 1). Intergroup comparison indicated that athletes with EIB had significantly lower post-exercise $\mathrm{FEV}_{1}$ than the athletes not showing EIB $(P<0.001)$ with a respective drop in $\mathrm{FEV}_{1}$ of $17.05 \pm 2.60$ and $3.99 \pm 3.77$ compared with values recorded prior to exercise.

The medical history questionnaire (Table 2 ) revealed that about $0.9 \%$ ( 1 out of 107 ) of the athletes had an allergy to some medication (Q 1) and almost 4.6\% (5 out of 107) had an allergy to insect bites or food (Q 2). Running causing chest symptoms (Q 8) was reported by $4.6 \%$ (5 out of 107) of the athletes, chest tightness by $4.6 \%$ (5 out of 107) and wheezing by $1.8 \%$ ( 2 out of 107). Among the 107 athletes, $1.8 \%$ ( 2 out of 107) reported having been previously diagnosed with asthma or EIB (Q 11).

The sensitivity and specificity of self-reported symptoms for EIB diagnosis were $35.71 \%$ and $96.77 \%$, respectively.

The agreement reached between the two methods (tests) using the kappa coefficient corresponds to $42 \%$ of the maximum.

\section{Discussion}

The major finding of the present study is that $13 \%$ (14 out of 107) of the Tunisian elite athletes fulfilled the diagnostic criteria of EIB despite only $1.8 \%$ of these athletes reporting having been previously diagnosed with EIB and taking prescribed medications on a permanent basis (steroids and anti-inflammatories). The use of a self-reporting respiratory symptoms questionnaire alone to evaluate asthma may lead

Table 2 Questions asked in the medical history questionnaire

\begin{tabular}{|c|c|c|}
\hline Question & Yes/total & $\%$ (out of 107 ) \\
\hline I. Are you allergic to any medicine (eg, aspirin, penicillin, sulfa)? & $\mathrm{I} / \mathrm{I} 07$ & 0.9 \\
\hline 2. Are you allergic to any insect bites/stings or do you have any food allergies? & $5 / 107$ & 4.7 \\
\hline 3. Do you take any medications (over the counter, herbs, supplements, vitamins)? & $8 / 107$ & 7.5 \\
\hline 4. Do you ever have itching of the nose or throat or sneezing spells? & $15 / 107$ & 14 \\
\hline 5. Do you ever have chest tightness? & $5 / 107$ & 4.7 \\
\hline 6. Do you ever have wheezing? & $2 / 107$ & 1.8 \\
\hline 7. Do you ever have itchy eyes? & $13 / 107$ & 12.2 \\
\hline 8. Does running ever cause chest tightness or cough or wheezing or prolonged shortness of breath? & $5 / 107$ & 4.7 \\
\hline $\begin{array}{l}\text { 9. Have you ever had chest tightness, cough, wheezing, asthma, or other chest (lung) problems, which made } \\
\text { it difficult for you to perform in sports? }\end{array}$ & $13 / 107$ & 12.2 \\
\hline $\begin{array}{l}\text { 10. Do you take any prescribed medications on a permanent or semi-permanent basis (steroids, birth } \\
\text { control pills, anti-inflammatories, antibiotics)? }\end{array}$ & $3 / 107$ & 2.8 \\
\hline II. Have you ever been told that you have (had) asthma or exercise-induced asthma? & $2 / 107$ & 1.8 \\
\hline 12. Do you have trouble breathing or do you cough during or after activity? & $2 / 107$ & 1.8 \\
\hline $\begin{array}{l}\text { 13. Have you ever missed school, work, or practice because of chest tightness or cough or wheezing } \\
\text { or prolonged shortness of breath? }\end{array}$ & $2 / 107$ & 1.8 \\
\hline 14. If you have been told you have asthma, what medications have you taken? & $2 / 107$ & 1.8 \\
\hline I5. Do you have or have you ever had lung disease (pneumonia)? & $3 / 107$ & 2.8 \\
\hline 16. List current medications. & $2 / 107$ & 1.8 \\
\hline
\end{tabular}


to a number of false negative and false positive diagnoses. Surprisingly, in the present study, very few athletes reported exercise-induced breathlessness, or symptoms of wheezing and chest tightness. This could be explained by "the fact that they do not perceive, do not recognize or do not want to recognize these respiratory sensations." Another possible reason for athletes ignoring this disorder is the fear of being eliminated from the team or not being selected for events.

Curiously, a significant number of nonasthmatic athletes experienced symptoms such as breathing difficulties on completion of the exercise, perhaps due to exercise intensity. However, the questions concerning respiratory symptoms did not allow a clear distinction to be made between asthma and its absence in athletes. In addition, a large proportion of athletes with EIB (9/14 [65\%]) indicated no respiratory symptoms associated with exercise. This could be because they did not recognize these respiratory sensations or that their asthma is so well controlled that the sensation of respiratory discomfort has gone. Another possible reason may be that these athletes try to ignore the condition though fear of being removed from the team or fear of not being selected for competition. As pointed out by Rundell et al ${ }^{11}$ the advantage of using questionnaires is that they recruit a large number of subjects, they save a lot of time, and they are less expensive compared to other means of diagnosis. However, the use of questionnaires may lead to unreliable diagnoses.

When we compared the prevalence of EIB in athletes obtained by subjective and objective methods, we noticed a dramatic difference. This same discrepancy, however, seems less marked among Olympians than other categories of athletes. Using subjective methods, Fitch, ${ }^{12}$ Voy, ${ }^{13}$ and Weiler and Ryan ${ }^{1}$ diagnosed asthma in 9.3\%, 11.2\%, and $15.3 \%$ of the same group of Summer Games Olympic athletes, respectively. Voy ${ }^{13}$ found an incidence of $14.2 \%$ using objective measures of EIB.

In this regard, Langdeau and Boulet ${ }^{14}$ and Melani et $\mathrm{al}^{15}$ indicated a large disparity between the two methods. Indeed, various studies have shown that the prevalence of EIB as determined by the questionnaire approach rarely exceeds $20 \%$, while this percentage is largely exceeded when the studies use means of group variables respiratory testing.

The sensitivity and specificity of self-reported symptoms for EIB diagnosis were $35.71 \%$ and $96.77 \%$, respectively. This indicates that the self-reported symptoms have been shown to have only a low sensitivity but high specificity for EIB in elite athletes, and are thus not recommended in the athlete with pure EIB. Rundell et $\mathrm{al}^{11}$ and Thole et $\mathrm{al}^{16}$ demonstrated a poor sensitivity $(<62 \%)$ and poor specificity $(<86 \%)$ of detection of respiratory symptoms in athletes with EIB. Our results are in accordance with others showing that EIB can be asymptomatic. ${ }^{17-19}$ Rundell et al ${ }^{18}$ found that at least one symptom is present in $91 \%$ of the athletes with EIB compared with only $48 \%$ of those without. In another study, ${ }^{11}$ the same authors found symptoms present in $61 \%$ of the athletes diagnosed as having asthma by lung function testing and in only $41 \%$ of those in whom the testing was negative.

Collectively, these studies show the unreliable nature of data obtained solely from questionnaires based on clinical symptoms for the assessment of EIB. Indeed, while questionnaires are useful tools in assessing the prevalence of respiratory symptoms in a population they do not take into account inter-individual differences in levels of perception of the symptoms or tolerance. These results also further explain why the use of a self-reporting questionnaire on respiratory symptoms to evaluate EIB may lead to a number of false negative and false positive diagnoses. ${ }^{11}$ According to Parsons, ${ }^{4}$ patient history and examination have limitations, the history often being nonspecific and the examination at rest being normal. There are also many conditions that mimic EIB presenting with similar symptoms, such as vocal cord dysfunction, cardiac arrhythmias, cardiomyopathies, and gastroesophageal reflux disease. In the present study, and surprisingly, very few athletes experienced exerciseinduced breathlessness, wheezing, or chest tightness. This could be explained by the fact that they do not perceive, do not recognize, or do not want to recognize these respiratory sensations. ${ }^{20}$ Another possible reason for the athletes to ignore this disorder is their fear of being eliminated from the team or not being selected for events. Kappa coefficient corresponds to $42 \%$ of the maximum, which indicates a moderate agreement between the two tests. Langdeau and Boulet $^{7}$ proposed a number of reasons for the discrepancy between objective and subjective test results, the first of which is that the increased incidence of heightened airway responses to inhaled constrictor agents observed in athletes may not only suggest the presence of an abnormal condition such as EIB, but could be the reflection of a "normal" response to a "supranormal" stimulus such as high-intensity exercise. It is possible, as previously suggested by Anderson and Holzer ${ }^{27}$ in a thorough review of exercise-induced bronchoconstriction, that the humidifying process taking place in the small airways, in response to exercise, could lead to airway edema and/or mucus secretion and that in combination with the "physiological" shortening of smooth muscles would amplify the airway narrowing with a resulting reduction in $\mathrm{FEV}_{1}$. The second factor is that high-intensity 
exercise causes asymptomatic airway hyper-responsiveness. Finally, the authors reported that the low incidence observed in athletes could also suggest that EIB is under-diagnosed when questionnaires are used, as they rely on self-reporting of symptoms that could be ignored or not well perceived by athletes. The phenomenon of under-recognition of EIB can also exist even with a physician-made diagnosis of EIB, as nonspecialist physicians rely primarily on respiratory symptoms or treatment trials to make the diagnosis of EIB.

One of the limitations of the present study is the absence of the eucapnic voluntary hyperventilation $(\mathrm{EVH})$ challenge test recommended by the International Olympics Committee and shown to have a high specificity for EIB. ${ }^{21} \mathrm{EVH}$ has also been shown to be more sensitive at detecting EIB than the methacholine challenge $e^{22}$ or field or laboratory-based exercise testing. ${ }^{21}$ EVH can also be performed successfully and safely to investigate EIB in patients other than elite athletes. ${ }^{23}$ Because of the wide differential diagnosis of exertional respiratory complaints, the diagnosis of EIB based on history and self-reported symptoms alone can only be considered as being inaccurate..$^{10,24,25}$ Should health care providers rely on history alone to make a diagnosis of EIB, evidence has shown that they will be mistaken in over $50 \%$ of cases. A second limitation is the number of subjects $(n=107)$, which is lower than the theoretical number needed to study the prevalence of EIB in the studied population. The formula ${ }^{11}$ yields a sample size of 138 , based on a $10 \%$ expected prevalence of EIB, a desired precision for the calculated results of 5\%, and a desired confidence level of $P<0.05$. The actual number of 107 provides only $77 \%$ of the optimal sample size.

We believe that athletes should be informed of the heightened risk of developing EIB, as a large number appear unaware that they are affected by the condition. Furthermore, to adequately assess airway function in athletes, particularly with regard to the potential risk of developing symptomatic asthma, objective methods are preferred. A heavier reliance on such methods should lead to a better identification of airway dysfunction in athletes with clinical suspicion of EIB.

\section{Conclusion}

The findings from this study show a discrepancy in the incidence of EIB among Tunisian athletes as detected by objective $(13 \%)$ and subjective $(1.8 \%)$ tests. We suggest that the presence of EIB can be difficult to diagnose clinically given the lack of specific symptoms and the frequent misinterpretation of vigorous exercise-induced manifestations.
Questionnaires are useful tools when evaluating the prevalence of respiratory symptoms in a population. However, they do not take into consideration the varying levels of perception and/or tolerance to symptoms among individuals. A complete history and physical examination should be performed on each athlete with respiratory complaints associated with exercise. However, because of the limitations and poor predictive value of patient history in the evaluation of EIB, objective diagnostic testing should be performed when EIB is suspected.

\section{Disclosure}

The authors report no conflicts of interest in this work.

\section{References}

1. Weiler JM, Ryan EJ 3rd. Asthma in United States Olympic athletes who participated in the 1998 Olympic Winter Games. J Allergy Clin Immunol. 2000;106(2):267-271.

2. Holzer K, Anderson SD, Douglass J. Exercise in elite summer athletes: challenges for diagnosis. J Allergy Clin Immunol. 2002;110(3): 374-380.

3. Tanaka J, Hasegawa T, Koya T, et al. Pulmonary function analysis of Japanese athletes: possibly even more asthmatics in the field. Allergol Int. 2010;59(1):53-57

4. Parsons JP. Current concepts in the diagnosis and management of exercise-induced bronchospasm. Phys Sportsmed. 2010;38(4):48-53.

5. Helenius IJ, Haahtela T. Allergy and asthma in elite summer sport athletes. J Allergy Clin Immunol. 2000;106(3):444-452.

6. Carlsen KH, Anderson SD, Bjermer L, et al. Exercise-induced asthma, respiratory and allergic disorders in elite athletes: epidemiology, mechanisms and diagnosis: Part I of the report from the Joint Task Force of the European Respiratory Society (ERS) and the European Academy of Allergy and Clinical Immunology (EAACI) in cooperation with GA2LEN. Allergy. 2008;63(4):387-403.

7. Langdeau JB, Boulet LP. Is asthma over- or under-diagnosed in athletes? Respir Med. 2003;97(2):109-114.

8. Weiler JM, Layton T, Hunt M. Asthma in United States Olympic athletes who participated in the 1996 Summer Games. JAllergy Clin Immunol. 1998;102(5):722-726.

9. American Thoracic Society. Standardization of spirometry, 1994 Update. Am J Respir Crit Care Med. 1995;152(3):1107-1136.

10. Crapo RO, Casaburi R, Coates AL, et al. Guidelines for methacholine and exercise challenge testing: 1999. This official statement of the American Thoracic Society was adopted by the ATS Board of Directors, July 1999. Am J Respir Crit Care Med. 2000;161(1):309-332.

11. Rundell KW, Im J, Mayers LB, Wilber RL, Szmedra L, Schmitz HR. Self-reported symptoms and exercise-induced asthma in the elite athlete. Med Sci Sports Exerc. 2001;33(2):208-213.

12. Fitch KD. Management of allergic Olympic athletes. J Allergy Clin Immunol. 1984;73(5 Pt 2):722-727.

13. Voy RO. The US Olympic Committee experience with exercise-induced bronchospasm, 1984. Med Sci Sports Exerc. 1986;18(3):328-330.

14. Langdeau JB, Boulet LP. Prevalence and mechanism of development of asthma and airway hyperresponsiveness in athletes. Sports Med. 2001;31(8):601-616.

15. Melani AS, Ciarleglio G, Pirrelli M, Sestini P. Perception of dyspnea during exercise-induced bronchoconstriction. Respir Med. 2003;97(3): 221-227.

16. Thole RT, Sallis RE, Rubin AL, Smith GN. Exercise-induced bronchospasm prevalence in collegiate cross-country runners. Med Sci Sports Exerc. 2001;33(10):1641-1646. 
17. Nystad W, Harris J, Borgen JS. Asthma and wheezing among Norwegian elite athletes. Med Sci Sports Exerc. 2000;32(2):266-270.

18. Rundell KW, Wilber RL, Szmedra L, Jenkinson DM, Mayers LB, Im J. Exercise-induced asthma screening of elite athletes: field versus laboratory exercise challenge. Med Sci Sports Exerc. 2000;32(2): 309-316.

19. Rundell KW, Spiering BA, Evans TM, Baumann JM. Baseline lung function, exercise-induced bronchoconstriction, and asthma-like symptoms in elite women ice hockey players. Med Sci Sports Exerc. 2004;36(3):405-410.

20. Turcotte H, Langdeau JB, Thibault G, Boulet LP. Prevalence of respiratory symptoms in an athlete population. Respir Med. 2003;97(8): 955-963.

21. Eliasson AH, Phillips YY, Rajagopal KR, Howard RS. Sensitivity and specificity of bronchial provocation testing. An evaluation of four techniques in exercise-induced bronchospasm. Chest. 1992;102(2): $347-355$.
22. Holzer K, Anderson SD, Douglass J. Exercise in elite summer athletes: challenges for diagnosis. J Allergy Clin Immunol. 2002;110(3): 374-380.

23. Brummel NE, Mastronarde JG, Rittinger D, Philips G, Parsons JP. The clinical utility of eucapnic voluntary hyperventilation testing for the diagnosis of exercise-induced bronchospasm. J Asthma. 2009; 46(7):683-686.

24. Parsons JP, Kaeding C, Phillips G, Jarjoura D, Wadley G, Mastronarde JG. Prevalence of exercise-induced bronchospasm in a cohort of varsity college athletes. Med Sci Sports Exerc. 2007;39(9):1487-1492.

25. Hallstrand TS, Curtis JR, Koepsell TD, et al. Effectiveness of screening examinations to detect unrecognized exercise-induced bronchoconstriction. J Pediatr. 2002;141(3):343-348.

26. Tabka Z, Hassayoune H, Guenard H, et al. Spirometric reference values in a Tunisian population. Tunis Med. 1995;73(2):125-131.

27. Anderson SD, Holzer K. Exercise-induced asthma: is it the right diagnosis in elite athletes? J Allergy Clin Immunol. 2000;106:419-428.
Open Access Journal of Sports Medicine

\section{Publish your work in this journal}

Open Access Journal of Sports Medicine is an international, peer-reviewed, open access journal publishing original research, reports, reviews and commentaries on all areas of sports medicine. The manuscript management system is completely online and includes a very quick and fair peer-review system.

\section{Dovepress}

Visit http://www.dovepress.com/testimonials.php to read real quotes from published authors. 